\title{
Adecuación y análisis de sensibilidad de un modelo para la estimación de la capacidad de carga del hábitat de venado cola blanca
}

\author{
Fitness and sensitivity analysis of a model to estimate carrying capacity \\ in the habitat from white-tailed deer

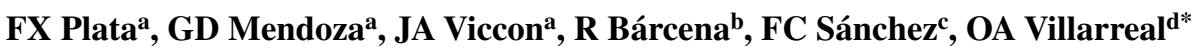 \\ aUniversidad Autónoma Metropolitana, Xochimilco, México.

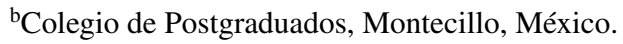 \\ ${ }^{\mathrm{c} C o l e g i o ~ d e ~ P o s t g r a d u a d o s, ~ S a n ~ L u i s ~ P o t o s i ́, ~ M e ́ x i c o . ~}$ \\ ${ }^{\mathrm{d} B e n e m e ́ r i t a ~ U n i v e r s i d a d ~ A u t o ́ n o m a ~ d e ~ P u e b l a, ~ P u e b l a, ~ M e ́ x i c o . ~}$
}

\begin{abstract}
SUMMARY
The aim of this study was to fit a model to estimate the carrying capacity $(K)$ for white-tailed deer and to perform a sensitivity analysis using data of a hunting ranch located in the semi-arid region of Aguascalientes, Mexico. The model had two main components, the first one calculates the metabolizable energy available in a given area from the accessibility of vegetal matter by strata (forbs, grasses, shrubs, trees and the total biomass), and the second component considered the energetic cost of the deer based on its physiological state, sex, approximated live weight and time of the year. The sensitivity analysis revealed that the variables with a higher impact on the value of change of $K(>5 \%)$ are the digestibility of the food, the total available biomass and the live weight of the animal. The lower impact on $K(<5 \%)$ is caused by the type of plant, their proportion in the site and the sex or the physiological state of the animal. It was possible to observe that the increase in the plant variables improves the carrying capacity whereas the increase in the animal energy requirements reduces it.
\end{abstract}

Palabras clave: capacidad de carga, modelos matemáticos, venado cola blanca, requerimientos energéticos.

Key words: carrying capacity, mathematical models, white-tailed deer, habitat, energetic requirements.

\section{INTRODUCCIÓN}

En México la cacería de venado cola blanca se practica legalmente en unidades de manejo para la conservación de la vida silvestre, las cuales son reguladas por la Secretaría del Medio Ambiente y Recursos Naturales ${ }^{1}$; dicha ley establece que, para el otorgamiento de los permisos de caza, se requiere un plan de manejo que incluye la estimación de la capacidad de carga $(K)$ y de la densidad poblacional de la especie silvestre que se va a aprovechar en la unidad.

Existen diversos métodos para determinar $K$ del hábitat del venado cola blanca en vida libre, pero uno de los más reportados en la literatura es el del aporte nutricional (McCall y col 1997), donde se relacionan los componentes nutricionales requeridos por el animal (materia seca, energía digestible, energía metabolizable y proteína cruda) con los kg de materia vegetal y/o los componentes nutricionales disponibles para el consumo animal (Mautz

\footnotetext{
Aceptado: 05.05.2011.

* mazamiztli@yahoo.com.mx

1 Ley General de Vida Silvestre (2007). Nueva Ley publicada en el Diario Oficial de la Federación el 3 de julio de 2000. Última reforma publicada DOF 01-02-2007. http://www.diputados.gob. $\mathrm{mx} /$ LeyesBiblio/pdf/146.pdf.
}

y col 1976, Mautz 1978, McLeod 1997, Bork y Werner 1999) asumiendo que toda la biomasa disponible es consumida por el venado, lo cual no es una suposición válida. Debido a que el venado cola blanca es un herbívoro con una alta tasa de fermentación ruminal prefiere alimentos de rápida fermentación y evita los alimentos altos en fibra o con una baja tasa de digestión ruminal (Van Soest 1994, 1996), lo que trae como consecuencia que sus hábitos alimentarios sean los de un ramoneador con una mayor preferencia hacia las herbáceas y arbustivas (Ramírez 2004). Varios estudios muestran que aun bajo condiciones de excelente pastizal los venados solo consumen alrededor de un $8 \%$ de gramíneas del total de su dieta (Hansen y col 1977, Stuth y Winward 1977, Bryant y col 1979, Stuth y Sheffield 2001), por lo que la diversidad y disponibilidad de plantas útiles para la alimentación del venado en un área dada es un componente que tendría que considerarse en la estimación de $K$.

Por otro lado, la estación del año modifica tanto la disponibilidad de alimento como la velocidad del viento y la temperatura ambiental alterando tanto la actividad física como los requerimientos energéticos del venado cola blanca (Moen 1976), ocasionando conductas estacionales y cambios en su metabolismo que modifican sus 
requerimientos energéticos (Robbins 1993). Actualmente, los requerimientos energéticos de los venados están definidos en una forma estática (NRC 2007) a pesar de que las fluctuaciones en los mismos por efecto de la época del año, del peso vivo y del estado fisiológico pueden ser determinados con el modelo de estimación del metabolismo en vida libre desarrollado por Moen (1978).

Los modelos de estimación de $K$ en función de la disponibilidad de nutrientes han sido evaluados por McCall y col (1997) quienes analizaron el aporte de diferentes compuestos nutricionales para la estimación de $K$. Posteriormente Plata y col (2011) evaluaron los modelos más sobresalientes de dichos autores y los compararon con otros modelos menos conocidos (presión de pastoreo y metabolismo ecológico). Estos modelos mostraron un buen acercamiento con la densidad de población; sin embargo, sus resultados muestran que sobrestiman la $K$ de un sitio.

La utilidad de los modelos matemáticos como herramienta para la toma de decisiones depende del grado de precisión y exactitud que tienen para predecir un resultado, por lo que estas variables requieren de una evaluación que puede ser utilizada como evidencia para su aceptación (Tedeschi 2006). Una de las estrategias más comunes para evaluar un modelo es el análisis de sensibilidad, el cual consiste en determinar la magnitud del cambio en la respuesta, al cambio de valores en los componentes del modelo (Vélez-Pareja 2003). El impacto puede ser negativo, cero o positivo y se expresa como porcentaje de cambio (Bossel 1994).

Considerando lo anteriormente expuesto, se plantearon dos objetivos en este trabajo: 1) Adecuar el modelo de metabolismo ecológico para estimar $K$ a partir tanto de la disponibilidad y las características nutritivas de los grupos vegetales presentes en una región dada, como de las preferencias alimenticias y el gasto energético en diferentes épocas del año estimado a partir del metabolismo en vida libre del venado y 2) Evaluar la relevancia de la inclusión de dichos factores a través de un análisis de sensibilidad.

\section{MATERIAL Y MÉTODOS}

\section{ORGANIZACIÓN DEL MODELO}

El diagrama del modelo se presenta en la figura 1. Se reconoce que la época del año modifica tanto la disponibilidad, tipos de forraje (Otto y col 2001), digestibilidad y energía metabolizable de los mismos (Clemente y col 2005), así como los requerimientos nutritivos del animal (NRC 2007). Estos factores interactúan para modificar el consumo voluntario del animal y su velocidad de crecimiento, todo lo cual impacta sobre $K$ de una región dada.

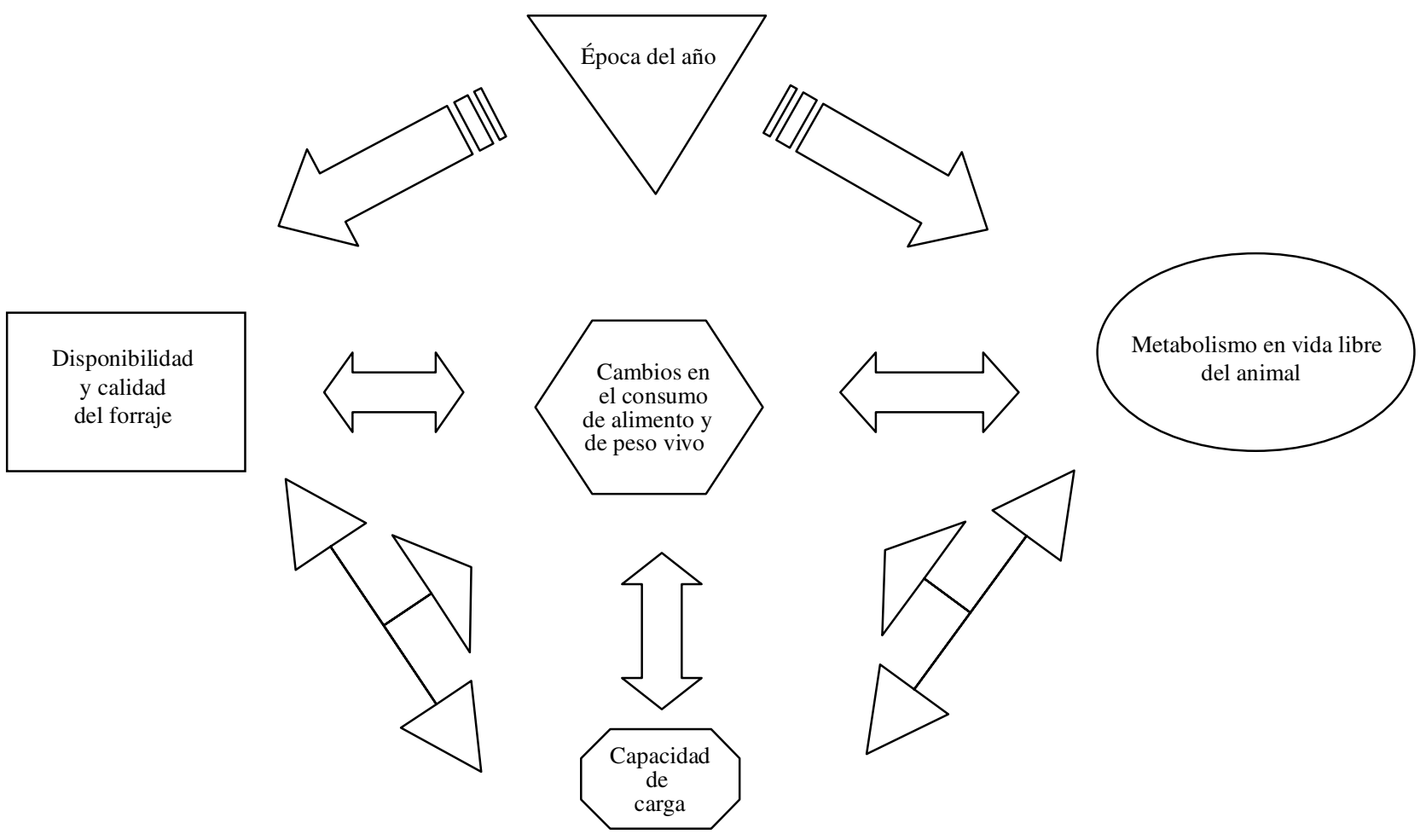

Figura 1. Modelo para determinar $K$ en venado cola blanca. Model to determine $K$ in white-tailed deer. 


\section{ÁREA DE ESTUDIO}

Se utilizó la información del muestreo de vegetación en un rancho cinegético localizado en el municipio de San José de Gracia, Aguascalientes, México. En este trabajo la vegetación se estimó mediante 19 sitios de muestreo con una extensión de $400 \mathrm{~m}^{2}$ (20 x 20 m) cada uno. La expresión matemática del modelo de metabolismo ecológico, las características nutricionales de la vegetación, clima y densidad de población que fueron utilizados para establecer las modificaciones a las características del modelo fueron publicados previamente (Clemente y col 2005, Plata y col 2011).

\section{COMPONENTES DEL MODELO MODIFICADO}

Para estimar la capacidad de carga, en este modelo se divide la energía metabolizable utilizable a partir de las arbóreas, arbustivas, herbáceas y gramíneas (considerando sus restricciones) entre el requerimiento energético de un animal de un peso y sexo dados durante un periodo $j$.

Matemáticamente, el modelo completo tiene la siguiente expresión:

$$
K=\left(\frac{E M_{u}+E M_{g}}{M E V_{p}}\right)
$$

$K=$ Capacidad de carga (venados/ha)

$E M_{u}=$ Energía metabolizable útil $(\mathrm{kcal} / \mathrm{kg})$

$E M_{g}=$ Energía metabolizable de gramíneas $(\mathrm{kcal} / \mathrm{kg})$

$M E V_{p}=$ Metabolismo en vida libre de un venado durante el periodo $\mathrm{p}$

Energía metabolizable útil $\left(E M_{u}\right)$. Debido a que el consumo máximo de forraje es inversamente proporcional al tamaño de los animales y a la selectividad que tiene el venado cola blanca, Stuth y Sheffield (2001) encontraron que la capacidad máxima de consumo del venado cola blanca en hábitats dominados por herbáceas es del 30\% y que la variación estimada del uso de este recurso para en el venado cola blanca es el 5\%, lo que sugiere que esta especie tiene un consumo equivalente al 35\% del hábitat, por lo que el modelo debe garantizar que el residuo de forraje es igual al 65\%; considerando lo anterior, la biomasa se ajusta en función de dicho coeficiente y se le denomina EM utilizable.

$$
E M_{u}=0,35 * E M_{a}
$$

Donde:

$E M_{u}=$ Energía metabolizable utilizable

0,35 = Capacidad máxima de consumo (Stuth y Sheffield 2001)

$E M_{a}=$ Energía metabolizable del área
Energía metabolizable del área. La EM que puede estar contenida en la biomasa vegetal de un área dada $(a)$ depende de la cantidad de biomasa $(B ; \mathrm{kg} / \mathrm{ha})$ de cada grupo vegetal (exceptuando gramíneas) y su $E M(\mathrm{kcal} / \mathrm{kg})$, por lo que la $E M_{a}$ es el resultado de la sumatoria de los productos de la $B$ de cada estrato vegetal por su respectiva $E M$ por el área total:

$$
E M_{a}=\sum_{1 \ldots n} B_{n} * E M_{n} *
$$

Donde:

$E M_{a}=$ Energía metabolizable del área

$\mathrm{n}=$ Grupo vegetal (arbórea, herbácea, arbustiva)

$\sum_{1 \ldots n} B_{n} * E M_{n} * a=$ Sumatoria del producto de la biomasa por la energía metabolizable de cada grupo vegetal por el área problema (ha)

Energía metabolizable de un grupo vegetal $n\left(E M_{n}\right)$. Para el cálculo de la $E M$ de un grupo vegetal $n$ se consideran los valores de energía digestible $(E D)$, la cual se estima con la información de calor de combustión y la digestibilidad in vitro promedio de la materia seca del mismo (Clemente y col 2005). Debido a que bajo condiciones de mantenimiento la eficiencia de conversión de la energía digestible a metabolizable es del 82\% (Ullrey 1970), el valor de $E D$ se multiplica por 0,82 . Por lo tanto, la energía metabolizable de un grupo vegetal $n$ es:

$$
E M_{n}=E B_{n} * D I V M S_{n} * 0,82
$$

Donde:

$E M_{n}=$ Energía metabolizable del grupo vegetal $n$

$E B_{n}=$ Calor de combustión del grupo vegetal ${ }_{n}$ $D_{V I V M} S_{n}=$ Digestibilidad in vitro del grupo vegetal $_{n}$ 0,82 = Eficiencia de conversión de $E D$ a $E M$

Energía metabolizable de gramíneas. Asumiendo que el consumo de gramíneas es reducido y se encuentra entre el dos y el ocho por ciento de la dieta total del venado (Arnold y Drawe 1979, Gallina 1993) la biomasa total de este grupo se condicionó a la presencia de arbustivas en la zona, por lo que la energía metabolizable aportada por las gramíneas en un área dada $\left(E M_{g}\right)$ es expresada como:

$$
E M g=\left(\frac{B h^{*} 0,08 * a * B_{g} * a}{B_{g} * a}\right) * E B_{g} * D I V M S_{g} * 0,82
$$

Donde:

$B_{h}=$ Biomasa total de herbáceas por ha

$B_{g}=$ Biomasa total de gramíneas por ha

$a=$ Área total (ha)

$E B_{g}=$ Energía bruta de las gramíneas

$D I V M S_{g}=$ Digestibilidad in vitro de las gramíneas 0,82 = Eficiencia de conversión de $E D$ a $E M$. 
Cuadro 1. Ecuaciones para la determinación estacional del metabolismo en vida libre del venado cola blanca.

Equations to predict seasonal changes in metabolism of white-tailed deer in wildlife.

Ecuación de Moen (1978) para hembras con cría

$$
\begin{array}{ll}
2 \cdot 56894 \ell^{0.19602(C)}\left(\begin{array}{l}
\left\{\left[1.0285 \operatorname{Sin}\left[\left(D J+170.053 \ell^{0.15488(C)}\right) 0.9863\right]+.0281\right][0.19+0.05(C)]\right\} \\
+[8.81-0.05(C)]
\end{array}\right) \\
.70\left\{\left[\left(\ell^{1.617+0.381 \ln (E E D)}\right) \cdot\{\operatorname{Sin}[(D J)(0.9863)+90] \cdot 0.1+0.90\}\right] 0.90\right\}^{0.75}
\end{array}
$$

Ecuación de Moen (1978) para machos

$\left.E M V=(\{[1.0285 \operatorname{Sin}[(D J) \cdot 0.9863]+239]+.0281\}+2.67) \cdot 70\left\{\left[\left(\ell^{1.617+0.381 \ln (E E D)}\right) \cdot\{\operatorname{Sin}[(D J)(0.9863)+90] \cdot 0.1+0.90\}\right] 0.90\right\}\right\}^{0.75}$

Ecuación de Moen (1978) modificada por Clemente (1984) para hembras.

$E M V=2.56894 \ell^{0.19602(C)}\left(\left\{\left[1.0285 \operatorname{Sin}\left[\left(D J+170.053 \ell^{0.15488(C)}\right) \cdot 0.9863\right]+281\right][0.19+0.05(C)]\right\}+[0.81-0.05(C)]\right) \cdot 70\left\{\left(\ell^{0.9928+\ln (P V)-.0771)}\right)\right\}^{0.75}$

Ecuación de Moen (1978) modificada por Clemente (1984) para machos

$E M V=(\{[1.0285 \operatorname{Sin}[(D J) \cdot 0.9863]+239]+.0281\}+2.67) \cdot 70\left\{\left(\ell^{0.9928+\ln (P V)-.0771}\right)\right\}^{0.75}$

$E M V=$ Es el requerimiento de $E M$ en vida libre del animal (hembras con cría). DJ = Es el día juliano en el cual se estima el gasto energético.

$\mathrm{C}=$ Es el número de crías. $\mathrm{PV}=$ Es el peso vivo del venado.

Requerimientos energéticos del venado. Para estimar el metabolismo en vida libre de un animal de un sexo y peso vivo determinado $(M E V)$ se utilizan las ecuaciones matemáticas desarrolladas por Moen (1978) y modificadas por Clemente (1984) cambiando la edad del animal por el peso vivo como una variable para estimar el peso metabólico del animal y en consecuencia el gasto de energía para mantenimiento. Dichas ecuaciones permiten estimar los cambios en los requerimientos energéticos del animal a lo largo del año, y modifican dicho gasto en función del sexo, peso vivo, estado físiológico del animal (lactancia) y número de crías. Tanto el modelo original de Moen (1976) como el modificado por Clemente (1984) se encuentran en el cuadro 1. El metabolismo en vida libre del venado $\left(M E V_{p}, \mathrm{kcal} / \mathrm{día}\right)$ se calcula utilizando una hoja de cálculo del programa Excel de Microsoft Office disponible en la plataforma de Bioeficencia ${ }^{2}$, con el cual se estima el requerimiento energético diario, por lo que el metabolismo energético de un periodo $(p)$ a evaluar está dado por:

$$
M E V_{p}=\sum_{1-i} M E V
$$

Donde:

$M E V=$ Metabolismo ecológico del venado (kcal/día) $j$ es el último día de un periodo dado.

2 Plata PF, GD Mendoza, E Riquelme. 2006. Metabolismo ecológico del venado cola blanca. En: www.bioeficiencia.avanzavet.com/index. php?option $=$ com_content \&task $=$ view $\&$ id $=84 \&$ Itemid $=1$, consultado el 12 de febrero de 2008.

\section{ANÁLISIS DE SENSIBILIDAD}

Se realizó un análisis de sensibilidad siguiendo la técnica descrita por Bossel (1994). Dentro de esta técnica, la variación en los datos se analiza individualmente, mediante la modificación de un valor a la vez, asumiendo que todos los demás permanecen sin alteración alguna (Vélez-Pareja, 2003). Para la generación de los parámetros iniciales en dicho análisis se utilizó información proveniente del rancho cinegético "El Altrialgo" localizado en el municipio de San José de Gracia, Aguascalientes, México. Dicha información fue publicada anteriormente (Plata y col 2011). Sin embargo, en el cuadro 2 se resumen los valores principales de la biomasa total por cada grupo vegetal así como los estimados de $E M_{n}, E M_{a}$ y la $E M_{u}$. También se presentan los valores de gasto energético por concepto de metabolismo en vida libre para un venado macho de $60 \mathrm{y}$ una hembra de $45 \mathrm{~kg}$ con una y dos crías. Dicha estimación se realizó considerando los días julianos 31, 59, 90, 120, $151,181,212,243,273,304,330$ y 365 que corresponden al día último de cada mes respectivamente y periodos de aproximadamente 30 días.

Utilizando como referencia los datos anteriores se definió un intervalo de valores normales para las variables evaluadas y se estimó $K$. Después, los valores biológicos de cada variable fueron sustituidos uno a uno, desde el valor menor al mayor, y se estimó el valor de cambio para cada incremento de la variable; dicho valor de cambio fue dividido entre el intervalo de cambio, de tal forma en que un valor cercano a cero indicó baja sensibilidad del modelo 
Cuadro 2. Biomasa y compuestos nutricionales disponibles por grupo vegetal y época del año, usados para estimar capacidad de carga.

Biomass and available nutrients by group of plants and season of the year used to estimate $K$.

\begin{tabular}{|c|c|c|c|c|c|}
\hline \multicolumn{6}{|c|}{ Materia Seca total (MS), kg/ha } \\
\hline Época del año ${ }^{1}$ & Gramíneas & Herbáceas & Arbóreas & Arbustivas & Total \\
\hline Verano & 617,89 & 114,72 & 137,17 & 16,86 & 886,64 \\
\hline Otoño & 311,99 & 114,53 & 72,94 & 12,22 & 511,68 \\
\hline Invierno & 162,21 & 195,31 & 118,39 & 20,61 & 496,52 \\
\hline \multicolumn{6}{|c|}{ Materia Seca Disponible, $\mathrm{kg} / \mathrm{ha}$} \\
\hline Verano & 216,26 & 40,15 & 48,01 & 5,90 & 310,32 \\
\hline Otoño & 109,20 & 40,09 & 25,53 & 4,28 & 179,09 \\
\hline Invierno & 56,77 & 68,36 & 41,44 & 7,21 & 173,78 \\
\hline \multicolumn{6}{|c|}{ Energía Metabolizable de los grupos vegetales $\left(E M_{n}\right)^{2}, \mathrm{kcal} / \mathrm{kg}$} \\
\hline Verano & 904.49 & 1913.64 & 1187.57 & 1385.74 & \\
\hline Otoño & 942.80 & 1994.44 & 1237.88 & 1443.23 & \\
\hline \multirow[t]{2}{*}{ Invierno } & 963.25 & 2037.71 & 1264.57 & 1476.16 & \\
\hline & $E M g, \mathrm{kcal} / \mathrm{kg}$ & Energía Metabol & e del área $(E M$ & $\mathrm{al} / \mathrm{kg}$ & \\
\hline Verano & $2.905,21$ & $142.023,7$ & & & \\
\hline Otoño & $3.023,74$ & $117.737,2$ & & & \\
\hline Invierno & $5.267,84$ & $202.345,0$ & & & \\
\hline \multicolumn{6}{|c|}{ Energía Metabolizable útil $\left(E M_{u}\right)^{4}, \mathrm{kcal} / \mathrm{kg}$} \\
\hline Verano & $144.928,93$ & & & & \\
\hline Otoño & $120.760,94$ & & & & \\
\hline Invierno & $207.612,86$ & & & & \\
\hline \multicolumn{6}{|c|}{ Metabolismo en vida libre del venado cola blanca } \\
\hline & 1 cría & 2 crías & Machos & & \\
\hline Verano & $318.058,714$ & $384.294,523$ & $361.771,367$ & & \\
\hline Otoño & $267.717,05$ & $251.400,831$ & $231.846,368$ & & \\
\hline Invierno & $399.590,394$ & $487.320,536$ & $504.129,479$ & & \\
\hline
\end{tabular}

${ }^{1}$ La duración estimada de los periodos de tiempo fue: Verano (91 días), Otoño (61 días) e Invierno (213 días).

${ }^{2}$ La DIVMS estuvo alrededor del 23 al 56\%, por lo que la EM por kg de MS fue de entre 1000 a $2000 \mathrm{kcal}$ por $\mathrm{kg}$.

${ }^{3}$ La sumatoria de la EM aportada por herbáceas, arbóreas y arbustivas.

${ }^{4}$ La sumatoria de $E M_{a}$ más $E M_{g}$.

a la variable. También se estimó el valor porcentual de cambio para cada variable.

\section{RESULTADOS}

En el cuadro 3 se presentan los resultados del análisis de sensibilidad del modelo para $K$ y en la figura 2 se observan los cambios estimados en $K$ de acuerdo al aumento de cada una de las variables contenidas en el modelo. En contraste con los modelos nutricionales de estimación de $K$, se puede observar que, exceptuando las gramíneas, el modelo es altamente sensible a los cambios en las características vegetales, las cuales mejoran la $K$ mientras que el aumento en el requerimiento energético del animal la reduce.
En la figura 3 se muestran los cambios en el requerimiento energético (metabolismo en vida libre) de animales con un peso determinado ( 45 y $60 \mathrm{~kg}$ ) pero considerando diferentes estados fisiológicos. Se puede observar que mientras durante el primer tercio del año los requerimientos energéticos son menores a lo largo del año se incrementa dicho requerimiento dependiendo del estado fisiológico del animal y del número de crías.

\section{DISCUSIÓN}

En este trabajo, el análisis de sensibilidad reveló que los cambios en la DIVMS o en la $E M_{g v}$ pueden incrementar $K$ de un sitio dado hasta en un $28,4 \%$. Dicho aumento es explicado como una consecuencia directa del aumento en la 
Cuadro 3. Análisis de sensibilidad de los componentes principales del modelo.

Sensibility analysis of the main components of the model.

\begin{tabular}{lcccccc}
\hline Variable & Promedio & Máximo & Mínimo & Intervalo & $\Delta K^{1}$ & $\begin{array}{c}\text { \% Promedio de } \\
\text { cambio }\end{array}$ \\
\hline Arbustivas, kg ms/ha & 16,41 & 20,61 & 12,22 & 1,398 & 0,0006 & 0,67 \\
Gramíneas, kg ms/ha & 390,05 & 617,89 & 162,21 & 75,95 & 0,0000 & 0,00 \\
Arbóreas, kg ms/ha & 105,055 & 137,17 & 72,94 & 10,71 & 0,0039 & 5,13 \\
Herbáceas, kg ms/ha & 154,65 & 195,31 & 114 & 13,55 & 0,0053 & 7,02 \\
MS total kg/ha & 661,37 & 961,37 & 361,37 & 100 & 0,0264 & 27,67 \\
DIVMS, \% & 41,55 & 60,7 & 22,4 & 6,38 & 0,0218 & 28,48 \\
Número de crías & 1 & 2 & 0 & 1 & $-0,0025$ & $-2,79$ \\
Peso vivo machos, kg & 45 & 75 & 15 & 10 & $-0,0243$ & $-11,64$ \\
Peso vivo hembras con una cría, kg & 45 & 75 & 15 & 10 & $-0,0202$ & $-11,64$ \\
Peso vivo hembras con dos crías, kg & 45 & 75 & 15 & 10 & $-0,0235$ & $-11,64$ \\
Día juliano & 196 & 361 & 31 & 55 & $-0,0027$ & $-3,01$ \\
Día juliano ajustado* & & & & & 0,0125 & 16,03 \\
\hline
\end{tabular}

1 Valor de cambio promedio en $K$.

* El ajuste se realizó elevando al cuadrado las diferencias de los intervalos y después se sacó la raíz cuadrada del promedio, de tal forma que los valores de cambio se convirtieron en valores absolutos.

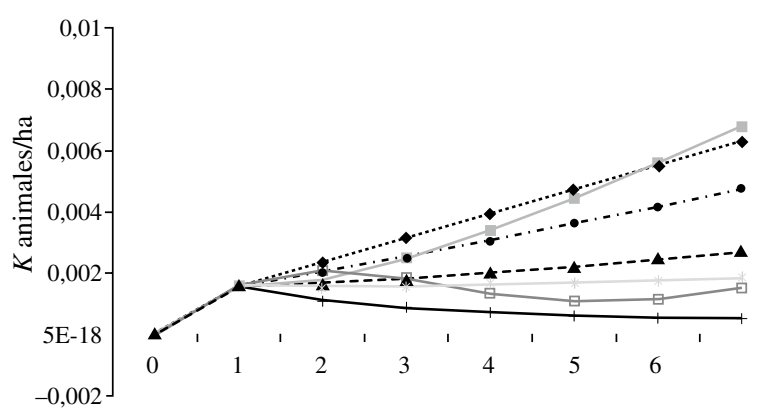

Intervalo de incremento de la variable

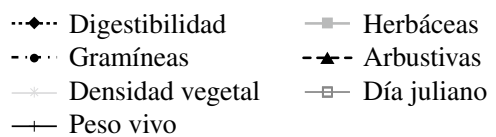

Figura 2. Efecto del incremento de las diferentes variables evaluadas mediante el análisis de sensibilidad en la capacidad de carga para venado cola blanca.

Effect of the increasing value of different variables evaluated in the sensibility analysis on the carrying capacity for white-tailed deer.

energía metabolizable del forraje. De acuerdo con Hanley (1997) la energía bruta de las diversas especies vegetales varía relativamente poco, por lo que la digestibilidad es la variable de mayor interés cuando se considera el aporte energético de un forraje. En las plantas silvestres la digestibilidad es variable, por lo que promover la vegetación con mayor digestibilidad podría favorecer tanto el crecimiento corporal como el número de venados.

Fryxell (1991) evaluando un modelo de consumo de energía digestible para rumiantes silvestres encontró que el aumento en la energía digestible del forraje permitía

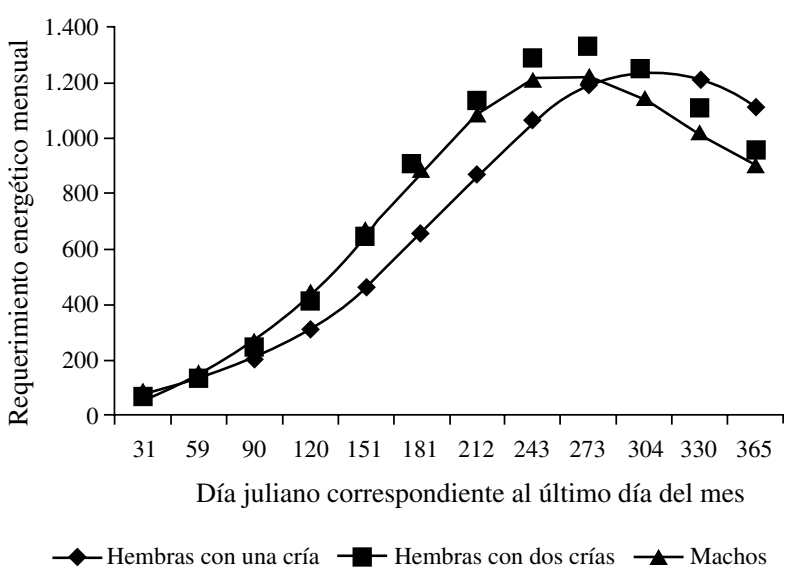

Figura 3. Efecto del peso vivo y el estado fisiológico del animal en el requerimiento energético del venado cola blanca.

Effect of live weight and physiological stage on the energy requirement of white-tailed deer.

un incremento en el número de animales por hectárea que se podían mantener, lo cual coincide con el análisis de sensibilidad de este trabajo. En el trabajo de dicho autor el aumento era dependiente de la tasa de crecimiento del forraje y del nivel de consumo de $E D$ del animal. Trabajos publicados por Bergman y col (2001) muestran que el consumo voluntario de los búfalos americanos (Bison bison) en vida libre estuvo asociado en forma positiva a los cambios en digestibilidad del forraje, de tal forma que el consumo de energía neta de ganancia se incrementó en forma lineal conforme aumentó la digestibilidad del forraje. 
También, el modelo desarrollado por Gordon e Illius (1994) utilizando diferentes tipos de rumiantes y herbívoros no rumiantes muestra la misma tendencia.

El análisis de sensibilidad muestra que el aumento en la biomasa total disponible tuvo un impacto importante $(27,67 \%)$ en $K$; este aumento puede ser explicado por la interacción entre la disponibilidad de alimento, tasa de consumo y respuesta funcional de los rumiantes, la cual ha sido establecida desde hace mucho tiempo (Gross y col 1993). En antílopes africanos, Brashares y Arcese (2002) encontraron que la abundancia y calidad del forraje eran determinantes en el grado de dispersión dentro y entre los grupos de hembras estudiados. Sus resultados muestran que el coeficiente de correlación entre el tamaño del grupo y la biomasa era de $0,55(\mathrm{P}<0,001)$. Farnsworth e Illius (1998) definieron un modelo de selección de dieta óptima para herbívoros mayores, el cual está fundamentado en la necesidad del animal de buscar y consumir alimento, lo cual ocurre en momentos separados, de tal forma que cuando la disponibilidad de forraje se reduce el tiempo de búsqueda aumenta, dicho comportamiento se explica en este modelo porque al aumentar la biomasa vegetal en forma independiente de la DIVMS, $K$ aumenta. Por el contrario, cuando la biomasa se reduce, los hábitos de consumo del venado cola blanca cambian junto con una reducción en la talla corporal de los mismos, lo cual tiene como finalidad poder mantener su condición reproductiva (Anouk Simard y col 2008).

El análisis de sensibilidad indicó que los cambios ocasionados por el incremento de $M S$ a partir de los diversos grupos vegetales (a excepción de las gramíneas) fueron directamente proporcionales a la materia seca disponible que aportaban para el mantenimiento de los animales, de tal forma que a mayor aporte de $M S$ mayor incremento en $K$. Estos grupos vegetales tenían un impacto de entre un 5 y $8 \%$ sobre $K$ dependiendo de la magnitud del aumento de la $M S$ y son menos relevantes que los cambios en la DIVMS o la biomasa total (figura 2). Sin embargo, no reflejó cambios de otra magnitud, debido a que la metodología de dicho análisis no permite la evaluación de la interacción nutriente y disponibilidad de $M S$. Así, la $D I V M S$ se analizó en forma separada de la disponibilidad de $M S$. Evidentemente, el impacto de las especies vegetales en $K$ está determinado tanto por el incremento de la biomasa como por el aporte de $E M_{g v}$ que realizan al área evaluada. Trabajos recientemente publicados (Codron y col 2007) mencionan que el aporte nutricional de las diversas especies vegetales cambia a lo largo del año dependiendo de la precipitación, por lo que su digestibilidad cambia en forma estacional; estos cambios en la digestibilidad están asociados a cambios en la relación de nitrógeno, fibra detergente neutro y lignina, por lo que dependiendo de la magnitud del cambio de estas variables, los valores nutritivos de las especies se modifican. También los datos de estos autores muestran que en algunas ocasiones las gramíneas tienen una mayor digestibilidad que los otros estratos vegetales, por lo que el aporte energético de los grupos puede ser similar, lo que ocasiona que su impacto sobre $K$ sea solo determinado por su incremento en la $M S$.

$\mathrm{El}$ análisis de sensibilidad sugiere que el modelo no modifica $K$ cuando se modifica la cantidad de gramíneas (cuadro 3), lo cual es explicado por el reducido aporte de las mismas al limitar su consumo a la disponibilidad de herbáceas. El trabajo de Gallina (1993) muestra que el venado cola blanca prefiere en su dieta hasta un $85 \%$ de arbóreas y arbustivas, mientras que las gramíneas ocuparon apenas un $2 \%$ de la dieta, la cual cambia de componentes vegetales de acuerdo a la época del año. Stuth y Sheffield (2001) estimaban que el consumo total de gramíneas para dicha especie era alrededor del 10\% y que el consumo de herbáceas o arbustivas dependía del predominio de estas especies en el sitio. Sin embargo, el trabajo de ReynaHurtado y Tanner (2005) muestra que el venado cola blanca modifica sus preferencias por el hábitat cuando se presentan alteraciones en el mismo.

En contraste a las variables anteriores, el análisis de sensibilidad muestra que el peso vivo tuvo un efecto negativo en $K$. Se sabe que el requerimiento energético de los venados se incrementa conforme se incrementa su peso metabólico (Moen 1978) y aunque dicho incremento no es lineal se ha demostrado que el aumento de tamaño implica un mayor gasto de energía total por día (Hanley 1982). El trabajo realizado por Owen-Smith (2008) muestra que el número de animales en un área dada se reduce cuando el peso vivo aumenta, lo cual se explica como un efecto directo que tienen los animales sobre la fisonomía, composición y funcionamiento de las plantas, las cuales pueden ser afectadas cuando los animales consumen diversas estructuras vegetales que garantizan la supervivencia de la planta (Skarpe y Hester 2008). No sólo el incremento en el consumo de nutrientes y los daños a la vegetación explican la reducción de $K$; debido a que el mayor consumo reduce las especies preferidas por el venado, la búsqueda de alimento subsecuente aumenta el tiempo dedicado a la misma y reduce el tiempo de ingesta (Hanley 1982). Etzenhouser y col (1998) mencionan que el venado modifica sus recorridos para encontrar determinado tipo de arbustivas que constituyen la base de su dieta. También los cambios de peso están relacionados con el ciclo reproductivo, alcanzando el peso máximo al inicio del otoño antes de que comience dicha actividad (Bartness y col 2002).

El día juliano parece tener un efecto reducido sobre $K$, lo cual se explica porque la época del año puede incrementar o disminuir la misma, de tal forma que el promedio de los cambios de esta variable tienden a cero; sin embargo, cuando se evalúa el impacto porcentual neto (independiente del efecto negativo o positivo) $K$ puede ser afectada hasta en un $15 \%$ por esta variable. Moen (1978) desarrolló este modelo partiendo de algunas premisas fundamentales, que incluyen la existencia de ciclos circadianos que se presentan a lo largo del año y que determinan la estacionalidad de muchas 
de las conductas de los animales; esos comportamientos cíclicos han sido reportados en aspectos reproductivos, aspectos hormonales, aspectos nutricionales y cambios de peso (Snyder y col 1983, Anderson y col 2003, Baker y col 2004). Los datos muestran que durante la época de invierno se observa una reducción en el requerimiento energético del venado; la cual está asociada a una reducción de la tasa metabólica basal, mientras que en el verano dicho requerimiento aumenta (Moen 1976, Arnold y col 2004). Este incremento en el requerimiento de energía está asociado a un mayor consumo de $M S$ por animal por día (Clauss y col 2003), lo cual reduce $K$.

El modelo mostró alta sensibilidad a los cambios en las características de los compuestos nutricionales de las plantas y a los cambios en la biomasa de las mismas, excluyendo a las gramíneas, todo lo cual modifica la capacidad de carga. Además, el modelo mostró una gran influencia al efecto de los cambios en los requerimientos energéticos del animal ocasionados por las modificaciones del peso vivo, el género y el estado fisiológico del animal. El modelo estima una utilización adecuada de gramíneas condicionada a la presencia de herbáceas y evita la sobrestimación de $K$ ocasionada por una alta cantidad de $M S$ de este grupo vegetal.

\section{RESUMEN}

Se adecuó un modelo para estimar la capacidad de carga $(K)$ de venados de cola blanca y se realizó un análisis de sensibilidad utilizando datos de un rancho cinegético localizado en la región semiárida de Aguascalientes, México. El modelo está compuesto por dos componentes, el primero estima la energía metabolizable disponible en un área dada a partir de la disponibilidad de materia vegetal por estratos (gramíneas, herbáceas, arbustivas, arbóreas y la biomasa total) y el segundo estima el gasto energético del venado basado en su estado fisiológico, sexo, peso vivo aproximado y época del año. El análisis de sensibilidad reveló que las variables con mayor impacto en el valor de cambio de $K(>5)$ son la digestibilidad del alimento, la biomasa total disponible y el peso vivo del animal. El menor impacto sobre $K(<5 \%)$ lo tuvieron los tipos de estratos vegetales, la proporción de los mismos en el sitio y el sexo o el estado fisiológico del animal. Se pudo observar que el aumento en las variables vegetales mejora la capacidad de carga del hábitat, mientras que el aumento en el requerimiento energético del animal reduce la misma.

\section{REFERENCIAS}

Anderson GM, SL Hardy, M Valent, HJ Billings, JM Connors, RL Goodman. 2003. Evidence that thyroid hormones act in the ventromedial preoptic area and the premammillary region of the brain to allow the termination of the breeding season in the ewe. Endocrinology 144, 2892-2901.

Anouk Simard M, SD Côté, RB Weladji, J Huot. 2008. Feedback effects of chronic browsing on life-history traits of a large herbivore. J Anim Ecol 77, 678-686.

Arnold Jr LA, L Drawe. 1979. Seasonal food habits of white-tailed deer in the South Texas Plains. J Range Manage 32, 175-178.

Arnold W, T Ruf, S Reimoser, F Tataruch, K Onderscheka, F Schober. 2004. Nocturnal hypometabolism as an overwintering strategy of red deer (Cervus elaphus). Am J Physiol Regu Integr Comp Physiol 286,174-181.

Baker DL, MA Wild, MM Connor, HB Ravivarapu, RL Dunn, TM Nett. 2004. Gonadotropin-releasing hormone agonist, a new approach to reversible contraception in female deer. J Wild Diseases 40,713-724.
Bartness TJ, GE Demas, CK Song. 2002. Seasonal changes in adiposity, the roles of the photoperiod, melatonin and other hormones and sympathetic nervous system. Exp Biol Med 227, 363-376.

Bergman CM, JM Fryxell, CC Gates, D Fortin. 2001. Ungulate foraging strategies, energy maximizing or time minimizing? J Anim Ecol 70, 289-300.

Bork WE, ST Werner. 1999. Viewpoint, Implications of spatial variability for estimating forage use. J Range Manage 52,151-156.

Bossel H. 1994. Modelling and Simulation. A.K. Peters Ltd., USA.

Brashares JS, P Arcese. 2002. Role of forage, habitat and predation in the behavioral plasticity of a small African antelope. J Anim Ecol 71, 626-638.

Bryant FC, MM Kothmann, LB Merrill. 1979. Diets of sheep, angora goats, spanish goats and white-tailed deer under excellent range conditions. J Range Manage 32, 412-417.

Clauss M, R Frey, B Kiefer, M Lechner-Doll, W Loehlein, C Polster, GE Rössner, WJ Streich. 2003. The maximum attainable body size of herbivorous mammals, morphophysiological constraints on foregut, and adaptations of hindgut fermenters. Oecologia 136, 14-27.

Clemen F, E Riquelme, GD Mendoza, R Bárcena, S González, R Ricalde. 2005. Digestibility of forage diets of white-tailed deer (Odocoileus virginuanus Hays) using different ruminal fluid inocula. J Appl Anim Res 27, 1-5.

Clemente SF. 1984. Utilización de la vegetación nativa del venado cola blanca (Odocoileus virginianus). Tesis de Maestría en Ciencias, Colegio de Postgraduados, Montecillo, Estado de México.

Codron D, JA Lee-Thorp, M Sponhelmer, J Codron. 2007. Nutritional content of savanna plants foods, implications for browser/grazer models of ungulate diversification. Eur J Wildl Res 53, 100-111.

Etzenhouser MJ, MK Owens, DE Spalinger, S Blake Murden. 1998. Foraging behavior of browsing ruminants in a heterogeneous landscape. Landscape Ecol 13, 55-64.

Farnsworth KD, AW Illius. 1998. Optimal diet choice for large herbivores, an extended contingency model. Functional Ecol 12, 74-81.

Fryxell JM. 1991. Forage quality and aggregation by large herbivores. The American Naturalist 138, 478-498.

Gallina S. 1993. White-tailed deer and cattle diets in La Michilia, Durango, Mexico. J Range Manage 46, 487- 492.

Gordon IJ, AW Illius. 1994. The functional significance of the browsergrazer dichotomy in African ruminants. Oecologia 98,167-175.

Gross JE, NT Hobbs, BA Wunder. 1993. Independent variables for predicting intake rate of mammalian herbivores, biomass density, plant density, or bite size? OIKOS 68, 75-91.

Hansen RM, RC Clark, W Lawhorn. 1977. Foods of wild horses, deer, and cattle in the Douglas mountain area, Colorado. J Range Manage 30, 116- 118.

Hanley TA.1982. The nutritional basis for food selection by ungulates. J Range Manage 35, 146-151.

Hanley TA. 1997. A nutritional view of understanding and complexity in the problem of diet selection by deer (Cervidae). Oikos 789, 209-218.

Mautz WW. 1978. Nutrition and carrying capacity. In: Schmidt J, Gilbert DL (eds). Big game of North America. Stackpole Books, Harrisburg, PA, USA, Pp 321-348.

Mautz WW, H Silver, JB Holter, HH Hayes, WE Urban. 1976. Digestibility and related nutritional data for seven northern deer browse species. $J$ Wild Manage 40, 630-683.

McCall TC, RD Brown, LC Bender. 1997. Comparison of techniques for determining the nutritional carrying capacity for white-tailed deer. J Range Manage 50, 33-38.

McLeod RS. 1997. Is the concept of carrying capacity useful in variable environments? Oikos 79, 529-542.

Moen AN. 1976. Energy conservation by white-tailed deer in the winter. Ecology 57, 192-198.

Moen AN. 1978. Seasonal changes in heart rates, activity, metabolism, and forage intake of white-tailed deer. J Wild Manage 42, 715-738. 
NRC, National Research Council. 2007. Energy. In: Committee on nutrient requirements of small ruminants (eds). Nutrient requirements of small ruminants (sheep, goats, cervids, and new world camelids). National Academic Press, Washington DC, USA, Pp 39-105.

Owen-Smith N. 2008. The comparative population dynamics of browsing and grazing ungulates. In: Gordon IJ, Prins HHT (eds). The Ecology of Browsing and Grazing. Vol 195. Springer-Verlag Berlin Heidelberg, Germany.

Plata PF, GD Mendoza, JA Viccon, R Bárcena, F Clemente. 2011. Comparación de métodos basados en los requerimientos nutricionales y disponibilidad de biomasa para estimar la capacidad de carga para venado cola blanca. Arch Med Vet 43, 41-50.

Ramírez GR. 2004. Nutrición del venado cola blanca. Universidad Autónoma de Nuevo León, Unión Ganadera Regional de Nuevo León y Fundación PRODUCE, Nuevo León, México.

Reyna-Hurtado R, GW Tanner. 2005. Habitat preferences of ungulates in hunted and nonhunted areas in the Calakmul Forest, Campeche, Mexico. Biotropica 37, 676-685.

Robbins CT. 1993. Energy requirements for maintenance. In: Robbins CT (ed). Wildlife feeding and nutrition. $2^{\text {nd }}$ ed. Academic Press Inc., San Diego, California, USA, Pp 114-174.

Skarpe C, A Hester. 2008. Plant Traits, Browsing and Grazing Herbivores and Vegetation Dynamics. In: Gordon IJ, Prins HHT (eds). The
Ecology of Browsing and Grazing. Vol. 195 Springer-Verlag Berlin Heidelberg, Germany.

Snyder DL, RL Cowan, DR Hagen, BD Schanbacher. 1983. Effect of pinealectomomy on seasonal changes in antler growth and concentrations of testosterone and prolactine in white-tailed deer. Biol Reprod 29, 63-71.

Stuth JW, AH Winward. 1977. Livestock-deer relations in the lodge pole pine-pumice region of central Oregon. J Range Manage 30,110-116.

Stuth JW, WJ Sheffield. 2001. Determining carrying capacity for combinations of livestock, white-tailed deer and exotic ungulates. Wildlife Management Handbook $V$ - A, 5 -12.

Tedeschi LO. 2006. Assessment of the adequacy of mathematical models. Agric Syst 89, 225-247.

Ullrey DE, WG Youatt, HE Johnson, LD Fay, BL Schoepke, WT Magee. 1970. Digestible and metabolizable energy requirements for winter maintenance of Michigan white-tailed does. J Wild Manage 34, 863-869.

Van Soest P. 1994. Evolution and role of ruminant. In: P Van Soest (ed). Nutritional ecology of the ruminant. O \& B Books Inc, Corvallis, Oregon, USA, Pp 1-13.

Van Soest P. 1996. Allometry and ecology of feeding behavior and digestive capacity in herbivores: A review. Zoo Biol 15, 455-479. 